\title{
Effect of membrane-bound complement regulatory proteins on tumor cell sensitivity to complement-dependent cytolysis triggered by heterologous expression of the $\alpha$-gal xenoantigen
}

\author{
YU WANG ${ }^{1 *}$, JUAN LIAO $^{1 *}$, YA-JUN YANG ${ }^{1}$, ZHU WANG $^{1}$, FENG QIN $^{2}$, \\ SHENG-MING ZHU ${ }^{3}$, HONG ZHENG ${ }^{1}$ and YAN-PING WANG ${ }^{1}$
}

${ }^{1}$ Laboratory of Molecular Diagnosis of Cancer, Clinical Research Center for Breast, West China Hospital, Sichuan University, Chengdu, Sichuan 610041; ${ }^{2}$ Basic Medical Faculty, Dali Medical College, Dali, Yunnan 671003;

${ }^{3}$ Department of Oncology, Affiliated Taihe Hospital, Yunyang Medical College, Shiyan, Hubei 442000, P.R. China

Received April 12, 2016; Accepted February 28, 2018

DOI: $10.3892 / \mathrm{ol} .2018 .8478$

\begin{abstract}
Engineering malignant cells to express a heterologous $\alpha$-gal antigen can induce heterograft hyperacute rejection, resulting in complement-dependent cytolysis (CDC) of tumor cells, which has been considered as a novel strategy for antitumor therapy. A549 cells engineered to express Gal $\alpha 1-3$ Gal $\beta 1-4 G 1 c N A c-R$ ( $\alpha$-gal) epitope exhibited strong resistance to CDC treated by normal human serum (NHS) in a previous study. We hypothesized that the expression of membrane-bound complement regulatory proteins (mCRPs) decay accelerating factor (CD55) and protectin (CD59) influenced the efficacy of the $\alpha$-gal/NHS-mediated antitumor effect to tumor cells in vitro. The present study confirmed that A549 cells expressed high levels of CD55 and CD59, whereas Lovo cells expressed relatively low levels of these proteins. A549 and Lovo cells transfected with plasmids containing or lacking the $\alpha$-gal epitope were evaluated for their susceptibility to CDC by NHS and detected using a trypan blue exclusion assay. $\alpha$-gal-expressing Lovo (Lovo-GT) cells were
\end{abstract}

Correspondence to: Professor Yan-Ping Wang, Laboratory of Molecular Diagnosis of Cancer, Clinical Research Center for Breast, West China Hospital, Sichuan University, 37 Guoxue Lane, Chengdu, Sichuan 610041, P.R. China

E-mail: wyanping@scu.edu.cn

*Contributed equally

Abbreviations: CDC, complement-dependent cytolysis; NHS, normal human serum; mCRPs, membrane-bound complement regulatory proteins; HAR, hyperacute rejection; MAC, membrane attack complex; mAbs, monoclonal antibodies; PI-PLC, phosphatidylinositol-specific phospholipase C; FCM, flow cytometry; PIEC, pig iliac arterial endothelial cells; ADCC, antibody-dependent cell-mediated cytotoxicity; MFI, mean fluorescence intensity

Key words: $\alpha$-gal epitope, membrane-bound complement regulatory proteins, complement-dependent cytolysis almost completely killed by $\alpha$-gal-mediated CDC following incubation with $50 \%$ NHS, whereas no cytolysis was observed in $\alpha$-gal expressing A549 (A549-GT) cells. Abrogating CD55 and CD59 function from A549-GT cells by various concentrations of phosphatidylinositol-specific phospholipase C (PI-PLC) or blocking antibodies increased the susceptibility of cells to $\mathrm{CDC}$, and the survival rate decreased significantly comparing to the controls $(\mathrm{P}<0.05)$. The findings of the present study indicated that using the $\alpha$-gal/NHS system to eliminate tumor cells via inducing the complement cascade reaction might represent a feasible approach for the treatment of cancer. However, high levels of mCRP expression may limit the efficacy of this approach. Therefore, an improved efficacy of cancer cell killing may be achieved by combining strategies of heterologous $\alpha$-gal expression and mCRP downregulation.

\section{Introduction}

The Gal $\alpha 1-3$ Gal $\beta 1-4 G l c N A c-R$ ( $\alpha$-gal) epitope structure is a carbohydrate epitope produced in virtually all non-primate mammal species, including pigs, rats, and New World monkeys (1). $\alpha$-gal is formed by the $\alpha 1,3$-galactosyltransferase $(\alpha-1,3 \mathrm{GT})$-mediated enzymatic transfer of a galactose molecule onto terminal $N$-acetyllactosamine on carbohydrate side chains of various glycoproteins and glycolipids (2,3). Owing to the inactivation of the $\alpha-1,3 \mathrm{GT}$ enzyme during evolution, the $\alpha$-gal epitope is absent in humans, apes, and Old World monkeys (4-7). Human blood therefore contains a large number of antibodies against the $\alpha$-gal epitope, including immunglobulin $\mathrm{G}(\operatorname{Ig} \mathrm{G})$, IgM, and $\operatorname{Ig} \mathrm{A}$, which are directly produced by B lymphocytes in response to constant stimulation from normal gastrointestinal flora $(8,9)$. These $\alpha$-gal-binding antibodies activate the classical complement pathway to induce a hyperacute rejection (HAR) $(10,11)$, which is largely responsible for the failure of xenotransplantation.

Engineering tumor cells to express the heterologous $\alpha$-gal antigen to induce host HAR against tumor cells is a novel antitumor strategy has been the focus of several prior studies (12-16). Indeed, certain studies observed a distinct complement-dependent cytolysis (CDC) of $\alpha$-gal-expressing 
tumor cells following treatment with normal human serum (NHS) $(12,16-18)$. However, it was recently identified that A549 tumor cells engineered to present $\alpha$-gal exhibited strong resistance to this $\alpha$-gal induced CDC by NHS $(19,20)$.

The complement system is a highly organized proteolytic cascade whose activation can be achieved by three different pathways (classical, mannan-binding lectin and alternative) depending on the initiating molecules. These three pathways all lead to the formation of the membrane attack complex (MAC), which mediates cell lysis (21). Normally, the activation of complement system is under the precise control of multiple factors, including the $\mathrm{C} 1$ esterase inhibitor, $\mathrm{C} 3 \mathrm{~b}$ inactivator and membrane-bound complement regulatory proteins (mCRPs), which avoid over-activation or excessive complement consumption to achieve a balance between immune activation and suppression (22). A substantial proportion of malignant tumor cells overexpress mCRPs, including membrane cofactor protein precursor (CD46), decay accelerating factor (CD55) $(23,24)$, and protectin (CD59) $(24,25)$. In a retrospective study evaluating the effectiveness of using the monoclonal antibody $(\mathrm{mAb})$ therapy trastuzumab against Her2-positive breast cancer, patients overexpressing CD55 or CD59 had a higher relapse rate and a shorter mean disease-free survival time than those with low expression of CD55 or CD59; the expression of CD46 had no effect on prognosis (26). In another study, it also suggested that CD55 and CD59 served roles in blocking trastuzumab-induced CDC in Her2-positive cells (27). Therefore, the overexpression of mCRPs may be a main factor behind the observed divergences in sensitivity to cell lysis by NHS among diverse $\alpha$-gal-expressing tumor cells

The present study examined whether expression levels of CD55 and CD59 in $\alpha$-gal-expressing cell lines influenced their CDC resistance. Following detection of CD55 and CD59 in various tumor cell lines by flow cytometry (FCM), Lovo colonic cancer cells which express low levels of CD55 and CD59, and A549 lung cancer cells which express high levels of CD55 and CD59, were selected for transfection with an $\alpha-1,3 \mathrm{GT}$ gene plasmid. CDC was observed in $\alpha$-gal-expressing Lovo and A549 cells by NHS treatment. We also evaluated the resistant effects of CD55 and CD59 in A549 cells via cleaving them by phosphatidylinositol-specific phospholipase $\mathrm{C}$ (PI-PLC) and blocking them by mAbs.

\section{Materials and methods}

Cell lines. Human A549, SPC-A-1, GLC-82, and LTPEP-a-2 lung cancer cells; Lovo colonic adenocarcinoma cells; SMMC-7721 hepatocarcinoma cells; SGC-7901 gastric cancer cells; MCF-7 and BT549 breast cancer cells were obtained from The Cell Bank of Type Culture Collection of the Chinese Academy of Sciences (Shanghai, China). Pig iliac arterial endothelial cells (PIEC) were kindly provided by the Key Laboratory of Transplant Engineering and Immunology, Sichuan University. PIEC, which naturally express high levels of $\alpha$-gal, were used as a positive control to assess complement activity in cytolysis assays.

Sera. Pooled NHS, used as the source of complement and anti- $\alpha$-gal specific antibodies provided by the Central Blood Bank of West China Hospital, was stored at $-80^{\circ} \mathrm{C}$ in aliquots until assayed. Use of the human serum samples in the study was approved by the Clinical Test and Biomedical Ethics Committee of West China Hospital, Sichuan University.

Antibodies and reagents. Fluorescein isothiocyanate (FITC)-conjugated mouse anti-human CD55 (cat. no. FHF055) and CD59 (cat. no. FHF059) mAbs were purchased from Beijing 4A Biotech Co., Ltd. (Beijing, China). The mAbs against CD55 (cat. no. SC-59092), CD59 (cat. no. SC-59095), and $\beta$-actin (cat. no. SC-47778) for western blotting were purchased from Santa Cruz Biotechnology, Inc. (Dallas, TX, USA). The horseradish peroxidase (HRP)-labeled goat anti-rat IgG (cat. no. SC-2030) and goat anti-mouse IgG (cat. no. SC-2031) Abs were purchased from Santa Cruz Biotechnology, Inc. The blocking mAbs against CD55 (cat. no. CBL511) was purchased from EMDMillipore (Billerica, MA, USA). The blocking mAbs against CD59 (cat. no. AB9182) was purchased from Abcam (Cambridge, UK).

FITC-BS-IB4 lectin (FITC-conjugated Griffonia simplicifolia isolectin B) which specifically binds $\alpha$-gal was purchased from Vector Laboratories, Inc. (Burlingame, CA, USA). The $100 \mathrm{U} / \mathrm{ml}$ PI-PLC and liposome transfection reagent kit Lipofectamine 2000 were purchased from Invitrogen (Thermo Fisher Scientific, Inc., Waltham, MA, USA).

Eukaryotic expression plasmids. The eukaryotic pCMV-GT $\alpha$-gal expression plasmid and the control p1-GT plasmid, in which the cytomegalovirus promoter did or did not regulate $\alpha-1,3 \mathrm{GT}$ gene expression, respectively, were successfully constructed in a preliminary study (28).

Detection of CD55 and CD59 expression by FCM. Cells were removed from the culture flask using $0.25 \%$ trypsin and $0.25 \%$ EDTA, and washed in $1 \%$ bovine serum albumin (BSA; Sigma-Aldrich; Merck KGaA, Darmstadt, Germany) diluted in PBS and centrifuged at $300 \mathrm{x}$ g for $10 \mathrm{~min}$. The cells were then suspended in $100 \mu \mathrm{l} 1 \% \mathrm{BSA}$ and incubated with $10 \mu \mathrm{l}$ FITC-CD55 or FITC-CD59mAbs for $30 \mathrm{~min}$ at $37^{\circ} \mathrm{C}$. FCM was performed using FACSAriaI and data were analyzed using FACSDiva 6.0 software (both from BD Biosciences, Franklin Lakes, NJ, USA).

Detection of CD55 and CD59 expression by western blotting. Cells in the logarithmic growth phase were harvested and lysed at $4^{\circ} \mathrm{C}$ in radioimmunoprecipitation lysis buffer (Beyotime Institute of Biotechnology, Shanghai, China). Total protein concentration was determined using a BCA kit (Beyotime Institute of Biotechnology). A total of $30 \mu \mathrm{g}$ protein from each sample was separated by $12 \%$ SDS-PAGE and transferred to polyvinylidene difluoride membranes. The membranes were blocked with $5 \%$ nonfat milk in PBS-Tween $(0.1 \%$ Tween in PBS). Membranes were incubated overnight with the primary antibodies against CD55 (1:400), CD59 (1:800) and $\beta$-actin $(1: 8,000)$ in $5 \%$ nonfat milk at $4^{\circ} \mathrm{C}$. After washed with PBS-Tween 10 min x 3 times, Membranes were incubated $2 \mathrm{~h}$ with HRP-labeled goat anti-mouse IgG (dilution, 1:7,000) or goat anti-rat IgG (dilution, 1:8,000) at room temperature. After washed, the bands were visualized using chemiluminescent HRP substrate (cat. no. WBKLS0100; EMDMillipore), and detected using the ChemiDocXRS system. Data was analyzed 
by QuantityOne software (Bio-Rad Laboratories, Inc., Hercules, CA, USA).

Establishing stable $\alpha$-gal-expressing cell lines. The pCMV-GT or the control p1-GT plasmids $0.8 \mu \mathrm{g}$ mixed with $2 \mu 1$ Lipofectamine2000 were diluted in $100 \mu 1$ Opti-MEM and transfected into the A549 and Lovo cell lines, then incubated for $6 \mathrm{~h}$. The transfected cells were further cultured in in RPMI-1640 medium containing 10\% fetal bovine serum for an additional $48 \mathrm{~h}$. The transfected cells were termed A549-GT ( $\alpha$-gal expressing A549), A549-V (control), Lovo-GT ( $\alpha$-gal expressing Lovo), and Lovo-V (control), respectively. The transfected cells were then transferred at a 1:10 dilution into a 6-well plate where stably transfected A549 and Lovo cells were selected following cultivation in the presence of G418.

Following selection, stably transfected cells expressing $\alpha$-gal were identified by direct immunofluorescence staining. A total of $50 \mu \mathrm{l}$ FITC-BS-IB4 lectin (1:50 dilution in RPMI-1640) per well was added into the transfected cells $\left(1 \times 10^{4}\right)$, which had been plated for $24 \mathrm{~h}$. After a 20 -min incubation in dark, the cells were analyzed under an inverted fluorescence microscope.

Analysis of $\alpha$-gal expression on stable transfected cells was also performed by FCM. A total of $1 \times 10^{6}$ cells from each cell line were incubated in $100 \mu$ l FITC-BS-IB4 lectin (1:50 dilution in $1 \%$ BSA-PBS) for $1.5 \mathrm{~h}$ at $4^{\circ} \mathrm{C}$ in dark. Following centrifugation at $300 \mathrm{x}$ g for $10 \mathrm{~min}$ and immersion in $1 \mathrm{ml}$ paraformaldehyde fixative solution (1\% BSA $+1 \%$ paraformaldehyde) for $30 \mathrm{~min}$ at $4^{\circ} \mathrm{C}$ in the dark, the cells were then resuspended in $300 \mu 1$ 1\% BSA-PBS and analyzed by FCM, according to the aforementioned method.

To determine $\alpha-1,3 \mathrm{GT}$ mRNA expression in transfected cells, total RNA was extracted using an RNeasy Mini kit (cat. no. 74104) from (QiagenGmbH, Hilden, Germany). First-strand cDNAs were synthesized from total RNA using 5X all-in-one RTMasterMix (G492; Applied Biological Materials, Inc., Richmond, BC, Canada). PCR was performed using Easy-load PCR Master Mix (cat. no. D7251; Beyotime Institute of Biotechnology) in iCycler (Bio-Rad Laboratories, Inc.). The PCR primer for $\alpha-1,3 \mathrm{GT}$ and GAPDH was synthesized by Sangon Biotech (Shanghai, China) as following: $\alpha-1,3 \mathrm{GT}$ fragment forward, 5'-TCAATGCTGCTTGTCTCA-3' and reverse, 5'-TAAGTGCCTTCCCATA-3'; GAPDH fragment forward, 5'-GTCAGTGGTGGACCTGACCT-3' and reverse, 5'-AGG GGAGATTCAGTGTGGTG-3'. The following thermocycling conditions were used: $94^{\circ} \mathrm{C}$ for $2 \mathrm{~min}$, followed by 33 cycles of $94^{\circ} \mathrm{C}$ for $30 \mathrm{sec}, 50^{\circ} \mathrm{C}$ for $30 \mathrm{sec}$, and $72^{\circ} \mathrm{C}$ for $2 \mathrm{~min}$, with a final extension step of $72^{\circ} \mathrm{C}$ for $10 \mathrm{~min}$. Amplification products were analyzed by $2 \%$ agarose gel electrophoresis.

Trypan blue exclusion assay for CDC induced by $\alpha$-gal-expressing cells. A549, A549-V, A549-GT, Lovo, Lovo-V, Lovo-GT and PIEC cells were resuspended to $1 \times 10^{6}$ per tube, and incubated with various dilutions of NHS ( 0 , 15,30 , and $50 \%$ ) in a total volume of $500 \mu \mathrm{l}$ for $1 \mathrm{~h}$ at $37^{\circ} \mathrm{C}$. Next, $50 \mu \mathrm{l}$ of each group of the incubated cells were mixed with the same volume of $0.4 \%$ trypan blue. The number of living/dead cells were counted in a hemocytometer under a light microscope, and the survival and lysis rates were calculated as follows: survival rate $(\%)=$ number of living cells/(number of living cells + number of dead cells) x100; lysis rate $=100 \%$-survival rate.

Evaluating susceptibility of $\alpha$-gal-expressing A549 cells to $C D C$ following pre-exposure to PI-PLC. PBS diluted PI-PLC to concentrations of $0.001,0.01,0.05,0.1,0.2$, or $0.5 \mathrm{U} / \mathrm{ml}$ were used to pre-treat A549, A549-V and A549-GT cells at $37^{\circ} \mathrm{C}$ for $1 \mathrm{~h}$. Cells were then incubated with $500 \mu 150 \%$ NHS, and assessed using a trypan blue exclusion assay, as aforementioned.

A concentration of $0.1 \mathrm{U} / \mathrm{ml}$ PI-PLC was selected to determine the specific cleavage effects of PI-PLC on membrane CD55 and CD59 at $37^{\circ} \mathrm{C}$ for $1 \mathrm{~h} . \mathrm{CD} 55$ and CD59 in A549, A549-V and A549-GT cells were tested by western blot analysis as aforementioned. Results were compared with those from cells that did not undergo PI-PLC treatment.

Following treatment with $0.1 \mathrm{U} / \mathrm{ml}$ PI-PLC, levels of CD55 and CD59 in A549-GT cells were assessed by FCM, and the results compared with those obtained prior to PI-PLC treatment.

Evaluating susceptibility of $\alpha$-gal-expressing A549 cells to CDC following blocking CD55 and CD59. A549, A549-V and A549-GT cells $\left(1 \times 10^{6}\right)$ were pre-incubated with $10 \mu \mathrm{g} / \mathrm{ml}$ anti-CD55, anti-CD59 or anti-CD55 together with anti-CD59 at room temperature for $10 \mathrm{~min}$, then $50 \%$ NHS was added to give a final volume of $500 \mu \mathrm{l}$ and cells were incubated at $37^{\circ} \mathrm{C}$ for $1 \mathrm{~h}$. Each sample was assessed using trypan blue exclusion assay, as aforementioned.

Statistical analysis. Results were expressed as the mean \pm standard deviation. Statistical significance was tested using an independent Student's t-test in two groups or one-way analysis of variance for experiments consisting of more than two groups, with a Student-Newman-Keuls test used as the post hoc test. $\mathrm{P}<0.05$ was considered to indicate a statistically significant difference. SPSS software 16.0 (SPSS, Inc., Chicago, IL, USA) was used.

\section{Results}

Expression of CD55 and CD59, detected by FCM. A number of cell lines were evaluated for their surface CD55 and CD59 protein levels by FCM. The results revealed that these cell lines expressed distinct levels of surface CD55 and CD59 (Fig. 1A). Specifically A549 cells expressed high levels of CD55 (mean fluorescence intensity $(\mathrm{MFI})=276 \pm 23$ ) and CD59 (MFI $629 \pm 42$ ), whereas Lovo cells expressed relatively low levels of CD55 (MFI=134 \pm 12 ) and CD59 (MFI=119 \pm 17 ). Since A549 and Lovo cells differed significantly in their expression of CD55 and CD59, Lovo cells were chosen as the ideal comparative cell line to assess whether CD55 and CD59 expression influenced the $\alpha$-gal-mediated cytolysis to compare with A549 cells in the present study.

Similar results were observed in western blot analysis (Fig. 1B). Comparing these two cell lines, the CD55 and CD59 protein level in A549 cells were evidently higher than Lovo cells.

Expression of $\alpha-1,3 G T$ mRNA in stably transfected A549 and Lovo cells. The results of the present study revealed that 
A

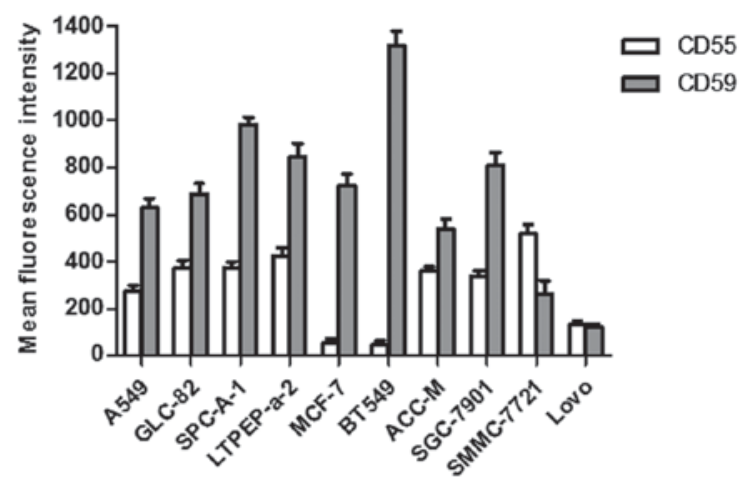

B

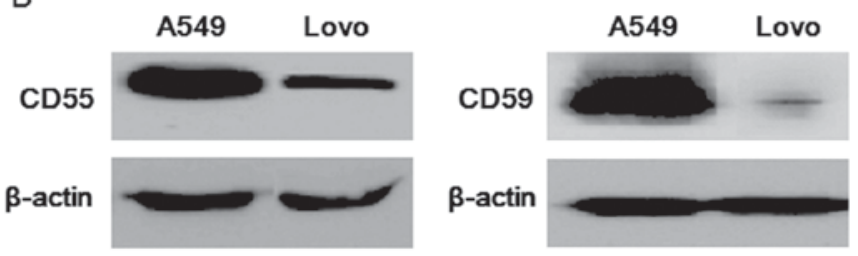

Figure 1. Expression of CD55 and CD59 in tumor cells. (A) Cell lines were incubated with fluorescein isothiocyanate-conjugated anti-human CD55 and CD59 monoclonal antibodies, and analyzed by flow cytometry. Error bars depict standard deviations. (B) The protein level of CD55 and CD59 in A549 and Lovo cells were detected by western blotting. $\beta$-actin protein levels served as the loading control. CD55, decay accelerating factor; CD59, protectin.

$\alpha-1,3 \mathrm{GT}$ mRNA was successful expressed in stably transfected A549-GT and Lovo-GT cells, but was absent from the control A549, A549-V, Lovo, and Lovo-V cells, as detected by agarose gel electrophoresis (Fig. 2A).

Expression of a-gal epitope on A549 and Lovo cells membranes. The stably transfected A549-GT and Lovo-GT cells exhibited high $\alpha$-gal epitope expression, as the observed staining was similar to that of PIEC positive-control cells. No fluorescent signals were observed on control A549, A549-V, Lovo, or Lovo-V cell membranes, which indicated that $\alpha$-gal epitope was specifically expressed in A549-GT and Lovo-GT cells (Fig. 2B).

The percentage of $\alpha$-gal-expressing cells in the stably transfected A549-GT and Lovo-GT cells, as well as in the PIEC cells were $80.1 \pm 3.2,95.4 \pm 5.2$, and $98.4 \pm 1.7 \%$, respectively, as detected by FCM (Fig. 2C).

Expression of CD55 and CD59 on $\alpha$-gal-expressing cells influences their sensitivity to CDC. A549, A549-V, A549-GT, Lovo, Lovo-V, Lovo-GT and PIEC cells were incubated with 0,15 , 30 or $50 \%$ NHS, and survival rates of these cells were calculated by trypan blue exclusion assay (Fig. 3A). A549, A549-V and A549-GT cells all exhibited resistance to CDC, and no evident changes were observed when the cells were exposed to a series of NHS concentrations. As the concentration of NHS increased from $0-50 \%$, the survival rate of Lovo-GT cells decreased gradually, reduced from 96.4 to $0.2 \%(\mathrm{P}<0.05)$. No significant cytolysis was observed in either Lovo or Lovo-V cells at any NHS concentration. In other words, $99.8 \%$ of Lovo-GT cells were killed when incubated with $50 \%$ NHS. The survival rate of Lovo-GT and PIEC positive-control cells exhibited similar concentration-dependency in NHS-induced CDC.
Increased susceptibility to CDC following cleavage of CD55 and CD59 in a-gal-expressing A549 cells. Incubating A549-GT cells with $0.001,0.01,0.05,0.1,0.2$, or $0.5 \mathrm{U} / \mathrm{ml}$ PI-PLC resulted in cell survival rates of 84.2, 72.3, 65.6, 54.9, 56.4 and $53.8 \%$, following treatment with 50\% NHS (Fig. 3B). A549-GT cells treated with PI-PLC of various dilutions experienced a significant decrease in survival rates $(\mathrm{P}<0.05)$. At a PI-PLC dose of $0.1 \mathrm{U} / \mathrm{ml}$, the survival rate of A549-GT cells decreased to $54.9 \%$, and did not decrease further at higher doses of PI-PLC. No significant cell death was observed in the control A549 or A549-V cells in the PI-PLC concentration series.

PI-PLC cleavage effect on CD55 and CD59 in A549-GT cells. CD55 and CD59 expression in A549, A549-V and A549-GT cells was almost completely abrogated following exposure to $0.1 \mathrm{U} / \mathrm{ml}$ PI-PLC (Fig. 4A).

The data obtained from FCM also confirmed these results, where CD55 (MFI=15 \pm 5 ) and CD59 (MFI=16 \pm 7) expression following PI-PLC treatment was significantly lower than that of CD55 (MFI=206 \pm 26$)$ and CD59 (MFI=755 \pm 84$)$ expression prior to PI-PLC treatment (Fig. 4B).

Blocking antibodies against CD55 and CD59 enhanced susceptibility to CDC in A549-GT cells. Following treatment with anti-CD55 or anti-CD59 antibodies alone, the survival rates of A549-GT cells significantly decreased to 80.5 and $49.3 \%$, respectively $(\mathrm{P}<0.05)$. Combination treatment with anti-CD55 and anti-CD59 further decreased the survival rate to $31.2 \%(\mathrm{P}<0.05)$. No significant decrease in cell survival was observed in the control A549 or A549-V cells in the anti-CD55-, anti-CD59- and anti-CD55 with anti-CD59-treated groups (Fig. 5).

\section{Discussion}

In xenotransplantation across species, such as pig to human, $\alpha$-gal expression on the cell surface of non-primate organs is the major xenoantigen responsible for HAR. A novel promising therapeutic approach can elicit the host anti- $\alpha$-gal immune response against the tumor cells to kill and/or inhibit tumor growth by expressing heterologous $\alpha$-gal antigen. Unfer et al (18) demonstrated that the co-incubation of $\alpha$-gal-expressing MC38 colon cancer cells with 50\% NHS led to $98 \%$ cell death. Other studies also found that transfection of the $\alpha-1,3 \mathrm{GT}$ gene into the A375 melanoma cells (12), MIA $\mathrm{PaCa}-2$ pancreatic cancer cells and Huh7 hepatocellular carcinoma cells (16), led to the differentiated susceptibility to CDC, compared with untransfected cells.

Although these studies $(12,16,18)$ revealed that the expression of the $\alpha$-gal xenoantigen sensitized tumor cells to NHS-induced cytolysis, certain other studies observed no statistical significance. Xing et al (14) examined the susceptibility of human SGC-7901, SPC-A-1 and A375 cells to NHS-mediated cytolysis with an $\alpha$-gal epitope expressed by adenoviral vector-mediated transfer of the pig $\alpha-1,3 \mathrm{GT}$ gene. No evident cell lysis was observed following incubation with 10, 20 or $40 \%$ NHS, as analyzed using a trypan blue exclusion assay. Jäger et al (29) revealed that $\alpha 1,3 \mathrm{GT}$-transfected HT1080 $\alpha$ tumor cells that expressed high levels of CD46, CD55, and 


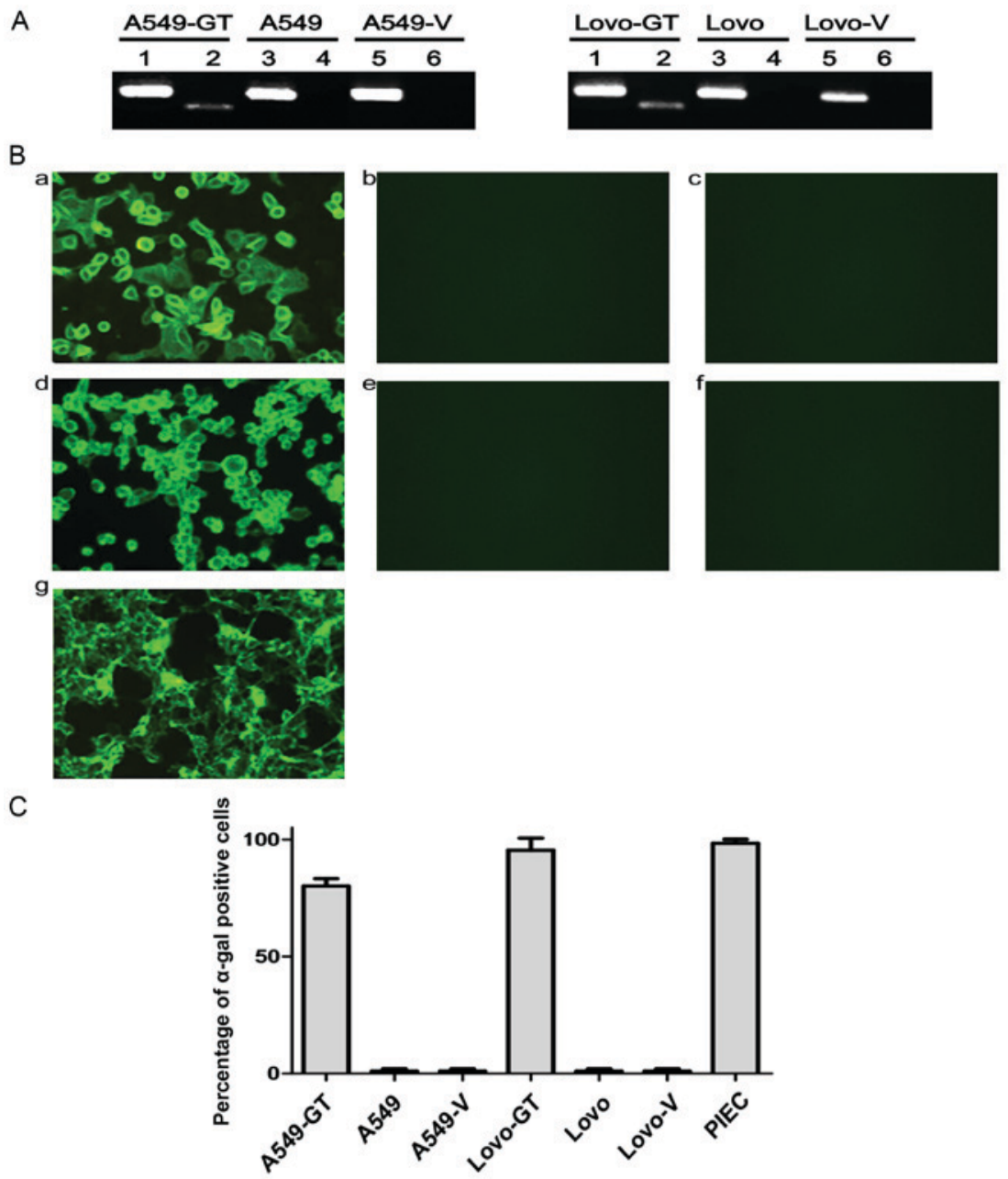

Figure 2. Establishing stable transfected $\alpha$-gal-expressing cell lines. (A) $\alpha-1,3$ GT mRNA expression in A549, A549-V, A549-GT, Lovo, Lovo-V and Lovo-GT cells were detected by reverse transcription-polymerase chain reaction. The amplified product of $\alpha-1,3 \mathrm{GT}$ was detected by agarose gel electrophoresis in lane 2,4 and 6 of the two gels. GAPDH was used as loading control in lane 1,3 and 5 of the two gels. (B) Expression of $\alpha$-gal epitope in each group of cells were detected by direct immunofluorescence (magnification, x200). FITC-conjugated BS-IB4 lectin staining was performed to probe $\alpha$-gal epitope. (B-a) A549-GT, (B-b) A549, (B-c) A549-V, (B-d) Lovo-GT, (B-e) Lovo, (B-f) Lovo-V and (B-g) positive control PIEC cells. (C) Expression of $\alpha$-gal epitope in each group of cells were stained with FITC-BS-IB4 lectin, then analyzed by flow cytometry. Error bars depict standard deviations. FITC, fluorescein isothiocyanate; $\alpha$-gal, Gal $\alpha 1$-3Gal/1-4GlcNAc-R; $\alpha$-1,3GT, $\alpha 1,3$-galactosyltransferase; PIEC, pig iliac arterial endothelial cells; A549-GT, $\alpha$-gal expressing A549; A549-V, control.

A

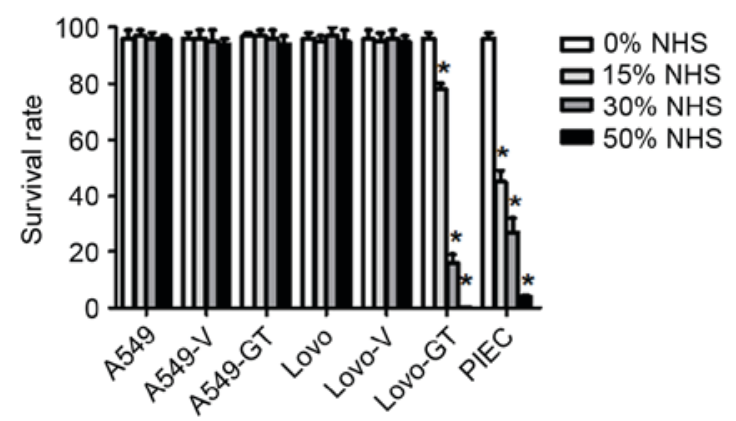

B

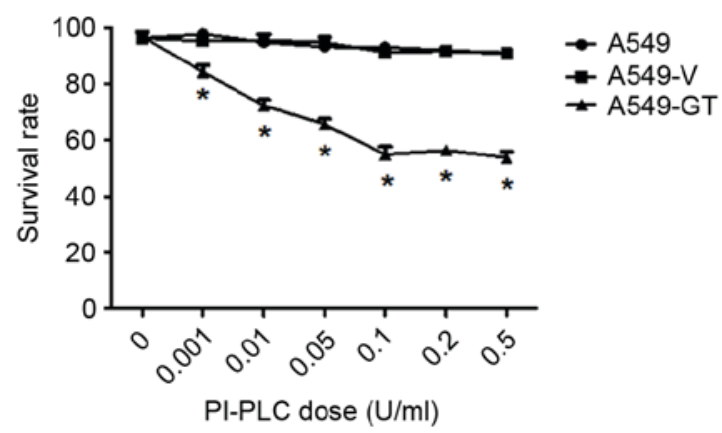

Figure 3. Expression of CD55 and CD59 on $\alpha$-gal-expressing cells influences their sensitivity to CDC. (A) A549, A549-V, A549-GT, Lovo, Lovo-V, Lovo-GT and positive control PIEC cells were incubated with various dilutions of NHS $(0,15,30,50 \%)$ and survival rates were analyzed by trypan blue staining. Error bars showed standard deviations (" $\mathrm{P}<0.05$ vs. the control). (B) A549, A549-V, A549-GT Cells were pre-treated with various concentrations of PI-PLC (0.001, $0.01,0.05,0.1,0.2$, or $0.5 \mathrm{U} / \mathrm{ml}$ ), incubated with $50 \% \mathrm{NHS}$, and survival rates were analyzed by trypan blue staining. Error bars showed standard deviations. "P $<0.05$, vs. the control. CD55, decay accelerating factor; CD59, protectin; NHS, normal human serum; PI-PLC, phosphatidylinositol-specific phospholipase C; A549-GT, $\alpha$-gal expressing A549; A549-V, control.

CD59 were not lysed by NHS, whereas cytolysis reached up to 75-80\% following PI-PLC-treatment.
More recently, the use of therapeutic antibodies is among the most active fields of cancer research. The binding of 
A

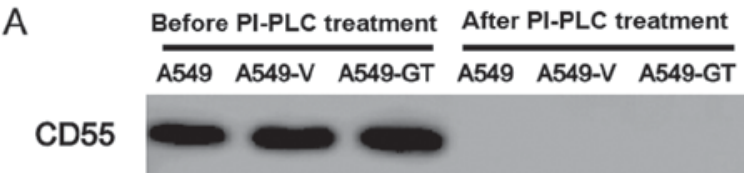

$\beta$-actin
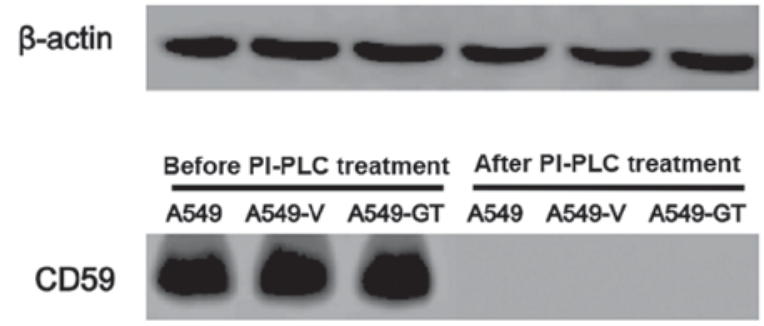

$\beta$-actin

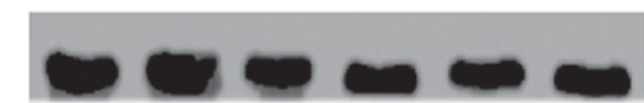

B

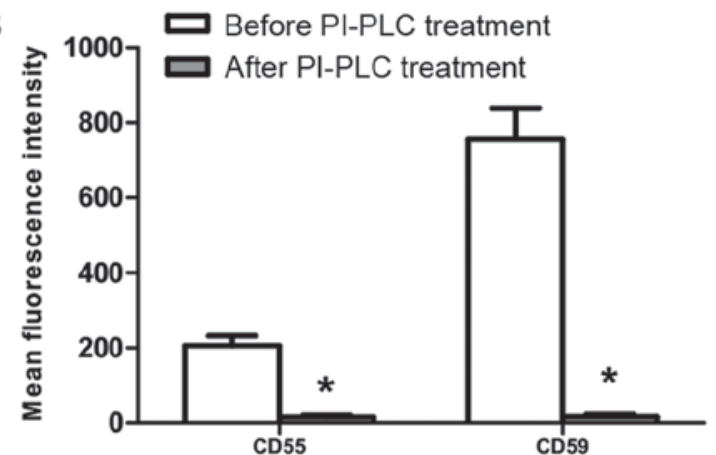

Figure 4. Effects of PI-PLC treatment on CD55 and CD59 protein level in A549-GT cells. (A) Following 0.1 U/ml PI-PLC treatment, CD55 and CD59 were tested by western blot in A549, A549-V, A549-GT, Lovo, Lovo-V and Lovo-GT cells, compared with that prior to PI-PLC treatment. (B) After 0.1 U/ml PI-PLC treatment, A549-GT cells was incubated with fluorescein isothiocyanate-conjugated anti-human monoclonal antibodies. CD55 and CD59 were analyzed by flow cytometry, compared with that prior to PI-PLC treatment. Error bars showed standard deviations. " $\mathrm{P}<0.05$ vs. the control. CD55, decay accelerating factor; CD59, protectin; PI-PLC, phosphatidylinositol-specific phospholipase C; A549-GT, $\alpha$-gal expressing A549; A549-V, control.

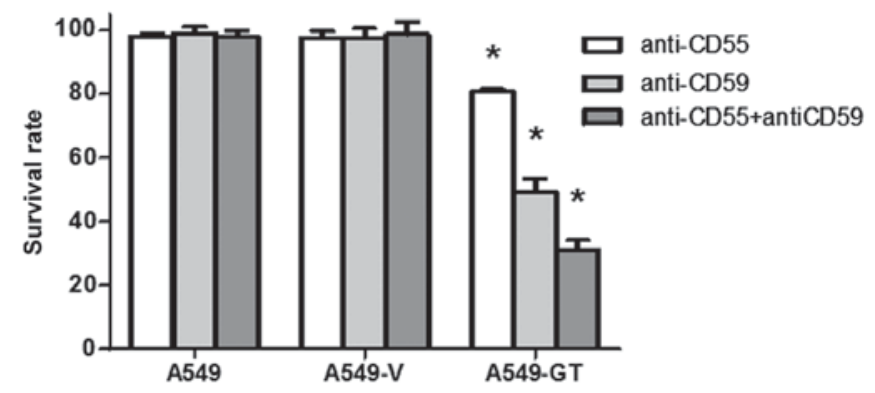

Figure 5. Effect of anti-CD55 and anti-CD59 on CDC in $\alpha$-gal-expressing cells. The A549, A549-V and A549-GT cells were pre-incubated with each antibodies (anti-CD55, anti-CD59 and anti-CD55 with anti-CD59) $(10 \mu \mathrm{g} / \mathrm{ml})$, then $50 \%$ normal human serum was added and the survival rates were calculated. Error bars showed standard deviations. "P<0.05 vs. the control. CD55, decay accelerating factor; CD59, protectin; $\alpha$-gal, Gal $\alpha 1-3 \mathrm{Gal} / 1$ 1-4GlcNAc-R; A549-GT, $\alpha$-gal expressing A549; A549-V, control.

tumor antigens and the corresponding antibodies can initiate the killing of tumor cells through CDC, antibody-dependent cell-mediated cytotoxicity (ADCC) and signal-pathway alteration (30). CDC is a mechanism that can lead to tumor cell lysis and also stimulate the adaptive immune response, as the fragments released upon CDC function to attract and activate immune cells (31). A number of types of tumor cells suppress the activation of the complement system by overexpressing mCRPs to evade complement attack (32-36). The main mCRPs are complement receptor 1 (CD35), CD46, CD55 and CD59 (37,38). CD55 is a membrane glycosyl-phosphatidyl inositol (GPI)-anchored glycoprotein that accelerates the decay of $\mathrm{C} 3$ convertases and C5 convertases, leading to the suppression of MAC activation (35). CD59 is also a small GPI-anchored glycoprotein that prevents the formation of a functional MAC by inhibiting the incorporation of multiple copies of $\mathrm{C} 9$ on the target cell membrane (37,39). In the present study, we hypothesized that CD59 and CD55 expression may have a role in the resistance of $\alpha$-gal-expressing A549 cells to $\alpha$-gal-mediated complement attack.

Other factors which may confer tumor resistance to anti- $\alpha$-gal Ab-mediated cytolysis were proposed in previous studies, such as blood type, anti- $\alpha$-gal Ab titers, and concentration of complementary factors. McMorrow et al (40) evaluated the anti- $\alpha$-gal antibodies levels in A, B, AB, and $\mathrm{O}$ serum samples using ELISA and flow cytometry, and observed a significant reduction in $\alpha$-gal reactive $\mathrm{IgG}$ in serum samples from $\mathrm{B}$-antigen-expressing donors (B, AB), comparing with non-B-antigen-expressing donors (A, O). Wang et al (41) revealed that the proportion of elderly individuals with low-affinity anti- $\alpha$-gal Abs is six-fold higher than that in young individuals, verifying that there might be an age-associated change in the affinity of anti- $\alpha$-gal antibodies. Koopmans et al (42) demonstrated that little $\alpha$-gal induced cytolysis occurred in pig mesencephalon cells cultured with NHS containing low anti- $\alpha$-gal IgM titers, whereas NHS containing 40 -fold higher anti- $\alpha$-gal IgM titers induced cell death in $65 \%$ of cells. In the present study, the influence of blood type, anti- $\alpha$-gal Ab titers, and concentration of complementary factors in the experiments was eliminated by: i) Using the same pooled serum (frozen in aliquots at $-80^{\circ} \mathrm{C}$ ) from healthy and young human donors; and ii) using the pig-derived PIEC cell line, which highly expresses $\alpha$-gal, as a positive control to assess antibodies and complement activity in cytolysis assays, where serum could be used only if the 50\% pooled NHS concentration killed $>95 \%$ of the PIEC cells.

In the present study, A549 and Lovo cells were chosen for further manipulation owing to their endogenous high and low expression of both CD55 and CD59, respectively, with the purpose of further confirmation of the role of mCRPs in the $\alpha$-gal/NHS CDC system. Lovo-GT cells were significantly more susceptible to NHS-mediated cytolysis, as almost all Lovo-GT cells were killed upon treatment with 50\% NHS. By contrast, A549-GT cells exhibited resistance to NHS-induced cytolysis with survival rates as high as $95 \%$. Consistent with prior speculation, in the $\alpha$-gal/NHS-mediated cytolysis system, tumor cells with low CD55 and CD59 expression were more susceptible to $\mathrm{CDC}$, whereas those with high expression of CD55 and CD59 might inhibit CDC.

The non-human PI-PLC enzyme can cleave the GPI-anchored mCRPs CD55 and CD59 at the cell membrane $(43,44)$. Therefore, a PI-PLC enzyme and corresponding blocking antibody were used to assess the suspected inhibitory effect of CD55 and CD59 on CDC. With 
an increase in PI-PLC dosage (0.001-0.1 U/ml), A549-GT survival rates significantly decreased from 96.9 to $54.9 \%$, which indicated that the cell sensitivity to NHS increased along with the enzyme concentration. When cells were treated with anti-CD55 or anti-CD59 blocking antibodies alone, the survival rate of A549-GT cells significantly decreased to 80.5 and $49.3 \%$, respectively. The combined use of anti-CD55 and anti-CD59 further decreased the survival rate to $31.2 \%$. The aforementioned results confirmed that the destruction of CD55 and CD59 functions on tumor cell membrane can reduce the resistance to $\alpha$-gal/NHS-mediated cell lysis.

In the present study, the killing effect of NHS induced CDC on A549-GT cells did not reach an ideal proportion, which may be a result of the inhibitory effects mediated by other complement regulatory proteins that were not assessed, such as soluble complement regulatory proteins factor $\mathrm{H}$, factor I, C1-inhibitor or mCRPs CD35, CD46. Other than by the aberrant expression of complement regulatory proteins, tumor cells can evade attack by the complement system via other mechanisms, including the repair of cell damage and preventing formation of the MAC. To make full use of the $\alpha$-gal/NHS therapy system to kill tumor cells, further in depth investigation into the possible influencing factors will be a necessity.

The in vitro cellular model presented in the current study may be difficult to adapt to in vivo studies owing to the lack of appropriate animal models. $\alpha-$ Gal was used as a target antigen to induce the complement cascade reaction to eliminate tumor cells, which may represent a prospective cancer therapy for human being in the future.

\section{Acknowledgements}

Not applicable.

\section{Funding}

The present study was supported by the National Natural Sciences Foundation of China (grant no. 30470762).

\section{Availability of data and materials}

The datasets used and/or analyzed during the current study are available from the corresponding author on reasonable request.

\section{Authors' contributions}

HZ and YPW conceived and designed the experiments. YW, JL, YJY, ZW, FQ and SMZ performed the experiments. YW and JL analyzed the data. YW and JL wrote the paper. All authors read and approved the final manuscript.

\section{Ethics approval and consent to participate}

The present study was approved by the Clinical Test and Biomedical Ethics Committee of West China Hospital, Sichuan University (2012 130).

\section{Consent for publication}

Not applicable.

\section{Competing interests}

The authors declare that they have no competing interests.

\section{References}

1. Kobayashi T and Cooper DK: Anti-Gal, alpha-Gal epitopes and xenotransplantation. Subcell Biochem 32: 229-257, 1999.

2. Eto T, Ichikawa Y, Nishimura K, Ando S and Yamakawa T: Chemistry of lipid of the posthemyolytic residue or stroma of erythrocytes. XVI. Occurrence of ceramide pentasaccharide in the membrane of erythrocytes and reticulocytes of rabbit. J Biochem 64: 205-213, 1968.

3. Galili U, Shohet SB, Kobrin E, Stults CL and Macher BA: Man, apes, and Old World monkeys differ from other mammals in the expression of alpha-galactosyl epitopes on nucleated cells. J Biol Chem 263: 17755-17762, 1988.

4. Koike C, Fung JJ, Geller DA, Kannagi R, Libert T, Luppi P, Nakashima I, Profozich J, Rudert W, Sharma SB, et al: Molecular basis of evolutionary loss of the alpha 1,3-galactosyltransferase gene in higher primates. J Biol Chem 277: 10114-10120, 2002.

5. Roy BB, Jinno-Oue A, Shinagawa M, Shimizu A, Tamura K Shimizu N, Tanaka A and Hoshino H: Isolation of the feline alpha1,3-galactosyltransferase gene, expression in transfected human cells and its phylogenetic analysis. J Exp Zool B Mol Dev Evol 306: 59-69, 2006.

6. Lanteri M, Giordanengo V, Vidal F, Gaudray P and Lefebvre JC: A complete alpha1,3-galactosyltransferase gene is present in the human genome and partially transcribed. Glycobiology 12 : 785-792, 2002.

7. Galili U: The alpha-gal epitope and the anti-Gal antibody in xenotransplantation and in cancer immunotherapy. Immunol Cell Biol 83: 674-686, 2005.

8. Peng SY and Wang WJ: The alpha-gal epitope (Gal alpha 1-3 Gal beta 1-4 GlcNAc-R) in xenotransplantation. Sheng Li Ke Xue Jin Zhan 34: 248-250, 2003 (In Chinese).

9. Galili U, Anaraki F, Thall A, Hill-Black C and Radic M: One percent of human circulating B lymphocytes are capable of producing the natural anti-Gal antibody. Blood 82: 2485-2493, 1993.

10. Kobayashi T: Problems and perspectives for clinical organ xenotransplantation. Nihon Jinzo Gakkai Shi 47: 83-93, 2005.

11. Walpen AJ, Mohacsi P, Frey C, Roos A, Daha MR and Rieben R: Activation of complement pathways in xenotransplantation: an in vitro study. Transpl Immunol 9: 271-280, 2002.

12. Link CJ Jr, Seregina T, Atchison R, Hall A, Muldoon R and Levy JP: Eliciting hyperacute xenograft response to treat human cancer: alpha $(1,3)$ galactosyltransferase gene therapy. Anticancer Res 18: 2301-2308, 1998.

13. Aubert M, Crotte C, Bernard JP, Lombardo D, Sadoulet MO and Mas E: Decrease of human pancreatic cancer cell tumorigenicity by alpha1,3galactosyltransferase gene transfer. Int J Cancer 107: 910-918, 2003.

14. Xing L, Xia GH,Fei J,Huang F and GuoLH: Adenovirus-mediated expression of pig alpha $(1,3)$ galactosyltransferase reconstructs Gal alpha $(1,3)$ gal epitope on the surface of human tumor cells. Cell Res 11: 116-124, 2001.

15. Deriy L, Chen ZC, Gao GP and Galili U: Expression of alpha-gal epitopes on HeLa cells transduced with adenovirus containing alpha1,3galactosyltransferase cDNA. Glycobiology 12: 135-144, 2002.

16. Yoshimura N, Sawada T, Furusawa M and Fuchinoue S: Expression of xenoantigen transformed human cancer cells to be susceptible to antibody-mediated cell killing. Cancer Lett 164: 155-160, 2001.

17. Takeuchi Y, Porter CD, Strahan KM, Preece AF, Gustafsson K, Cosset FL, Weiss RA and Collins MK: Sensitization of cells and retroviruses to human serum by (alpha 1-3) galactosyltransferase. Nature 379: 85-88, 1996.

18. Unfer RC, Hellrung D and Link CJ Jr: Immunity to the alpha $(1,3)$ galactosyl epitope provides protection in mice challenged with colon cancer cells expressing alpha $(1,3)$ galactosyl-transferase: A novel suicide gene for cancer gene therapy. Cancer Res 63: 987-993, 2003.

19. LiuM,ZhuSM,ZhengH,Wang Y,WangZ,Yang YJ,WuYX,Zeng YZ and Wang YP: Cloning of splicing variants of alpha1,3-galactosyltransferase cDNA of Chinese Banna Minipig inbred line and its expression in human cells. Sichuan Da Xue Xue Bao Yi Xue Ban 43: 145-150, 2012. 
20. Qin F, Zhu SM, Zheng H, Wang Z, Wang Y, Zuo Y, Chen J, Sun WL and Wang YP: Establishment and identification of human lung adenocarcinoma cell line stably expressing the alpha1, 3-galaetosyltransferase gene from pig. Sichuan Da Xue Xue Bao Yi Xue Ban 41: 194-198, 2010.

21. Bajic G, Degn SE, Thiel S and Andersen GR: Complement activation, regulation and molecular basis for complement-related diseases. EMBO J 34: 2735-2757, 2015.

22. Jurianz K, Ziegler S, Donin N, Reiter Y, Fishelson Z and Kirschfink M: K562 erythroleukemic cells are equipped with multiple mechanisms of resistance to lysis by complement. Int J Cancer 93: 848-854, 2001.

23. Loberg RD, Day LL, Dunn R, Kalikin LM and Pienta KJ: Inhibition of decay-accelerating factor (CD55) attenuates prostate cancer growth and survival in vivo. Neoplasia 8: 69-78, 2006

24. Ziller F, Macor P, Bulla R, Sblattero D, Marzari R and Tedesco F: Controlling complement resistance in cancer by using human monoclonal antibodies that neutralize complement-regulatory proteins CD55 and CD59. Eur J Immunol 35: 2175-2183, 2005.

25. Treon SP, Mitsiades C, Mitsiades N, Young G, Doss D, Schlossman R and Anderson KC: Tumor cell expression of CD59 is associated with resistance to CD20 Serotherapy in patients with B-cell malignancies. J Immunother (1991) 24: 263-271, 2001.

26. Liu M, Yang YJ, Zheng $\mathrm{H}$, Zhong XR, Wang Y, Wang Z, Wang YG and Wang YP: Membrane-bound complement regulatory proteins are prognostic factors of operable breast cancer treated with adjuvant trastuzumab: A retrospective study. Oncol Rep 32: 2619-2627, 2014.

27. Wang Y, Yang YJ, Wang Z, Liao J, Liu M, Zhong XR, Zheng H and Wang YP: CD55 and CD59 expression protects HER2 overexpressing breast cancer cells from trastuzumab-induced complement-dependent cytotoxicity. Oncol Lett 14: 2961-2969, 2017.

28. Zhu S, Wang Y, Zheng H, Cheng J, Lu Y, Zeng Y, Wang Y and Wang Z: Cloning of Chinese Banna minipig inbred-line alpha1,3-galactosyltransferase gene and construction of its recombinant eukaryotic expression vector. Sheng Wu Yi Xue Gong Cheng Xue Za Zhi 26: 360-365, 2009.

29. Jäger U, Takeuchi $Y$ and Porter $C$ : Induction of complement attack on human cells by $\mathrm{Gal}($ alpha1,3) Gal xenoantigen expression as a gene therapy approach to cancer. Gene Ther 6: 1073-1083, 1999.

30. Hendriks D, Choi G, de Bruyn M, Wiersma VR and Bremer E: Antibody-based cancer therapy: successful agents and novel approaches. Int Rev Cell Mol Biol 331: 289-383, 2017.

31. Shuptrine CW, Surana R and Weiner LM: Monoclonal antibodies for the treatment of cancer. Semin Cancer Biol 22: 3-13, 2012.

32. Hakulinen J, Junnikkala S, Sorsa T and Meri S: Complement inhibitor membrane cofactor protein (MCP; CD46) is constitutively shed from cancer cell membranes in vesicles and converted by a metalloproteinase to a functionally active soluble form. Eur J Immunol 34: 2620-2629, 2004.

33. Bellone S, Roque D, Cocco E, Gasparrini S, Bortolomai I, Buza N, Abu-Khalaf M, Silasi DA, Ratner E, Azodi M, et al: Downregulation of membrane complement inhibitors CD55 and CD59 by siRNA sensitises uterine serous carcinoma overexpressing Her2/neu to complement and antibody-dependent cell cytotoxicity in vitro: Implications for trastuzumab-based immunotherapy. Br J Cancer 106: 1543-1550, 2012.
34. Kesselring R, Thiel A, Pries R,Fichtner-Feigl S, Brunner S, Seidel P, Bruchhage KL and Wollenberg B: The complement receptors CD46, CD55 and CD59 are regulated by the tumour microenvironment of head and neck cancer to facilitate escape of complement attack. Eur J Cancer 50: 2152-2161, 2014.

35. Devarapu SK, Mamidi S, Plöger F, Dill O, Blixt O, Kirschfink M and Schwartz-Albiez R: Cytotoxic activity against human neuroblastoma and melanoma cells mediated by IgM antibodies derived from peripheral blood of healthy donors. Int J Cancer 138: 2963-2973, 2016.

36. Fishelson Z, Donin N, Zell S, Schultz S and Kirschfink M: Obstacles to cancer immunotherapy: expression of membrane complement regulatory proteins (mCRPs) in tumors. Mol Immunol 40: 109-123, 2003.

37. Gorter A and Meri S: Immune evasion of tumor cells using membrane-bound complement regulatory proteins. Immunol Today 20: 576-582, 1999.

38. Mamidi S, Cinci M, Hasmann M, Fehring V and Kirschfink M: Lipoplex mediated silencing of membrane regulators (CD46, CD55 and CD59) enhances complement-dependent anti-tumor activity of trastuzumab and pertuzumab. Mol Oncol 7: 580-594, 2013.

39. Loberg RD, Wojno KJ, Day LL and Pienta KJ: Analysis of membrane-bound complement regulatory proteins in prostate cancer. Urology 66: 1321-1326, 2005.

40. McMorrow IM, Comrack CA, Nazarey PP, Sachs DH and DerSimonian H: Relationship between ABO blood group and levels of Gal alpha,3Galactose-reactive human immunoglobulin G. Transplantation 64: 546-549, 1997.

41. Wang L, Anaraki F, Henion TR and Galili U: Variations in activity of the human natural anti-Gal antibody in young and elderly populations. J Gerontol A Biol Sci Med Sci 50: M227-M233, 1995.

42. Koopmans J, de Haan A, Bruin E, van der Gun I, van Dijk H, Rozing J, de Leij L and Staal M: Individual human serum differs in the amount of antibodies with affinity for pig fetal ventral mesencephalic cells and the ability to lyse these cells by complement activation. Cell Transplant 13: 631-637, 2004.

43. White MJ, Boyd JM, Horswill AR and Nauseef WM: Phosphatidylinositol-specific phospholipase C contributes to survival of Staphylococcus aureus USA300 in human blood and neutrophils. Infect Immun 82: 1559-1571, 2014.

44. Cummerson JA, Flanagan BF, Spiller DG and Johnson PM: The complement regulatory proteins CD55 (decay accelerating factor) and CD59 are expressed on the inner acrosomal membrane of human spermatozoa as well as CD46 (membrane cofactor protein). Immunology 118: 333-342, 2006.

This work is licensed under a Creative Commons Attribution-NonCommercial-NoDerivatives 4.0 International (CC BY-NC-ND 4.0) License. 